\title{
DataMoves: Entangling Data and Movement to Support Computer Science Education
}

Justas Brazauskas

j.brazauskas@ucl.ac.uk

UCL Interaction Centre, University

College London

London, UK

Rebecca Evans

pellensemble@gmail.com

Pell Ensemble

London, UK
Susan Lechelt

susan.lechelt@ed.ac.uk

Institute for Design Informatics,

University of Edinburgh

Edinburgh, UK

Su Adams

su@ucantoo.org.uk

U Can Too

Kent, UK
Ethan Wood

ethan.wood.18@ucl.ac.uk

UCL Interaction Centre, University

College London

London, UK

Emma McFarland

emma@emc-arts.co.uk

studio eMc

Devon, UK
Nicolai Marquardt

n.marquardt@ucl.ac.uk

UCL Interaction Centre, University

College London

London, UK
Yvonne Rogers

y.rogers@ucl.ac.uk

UCL Interaction Centre, University

College London

London, UK
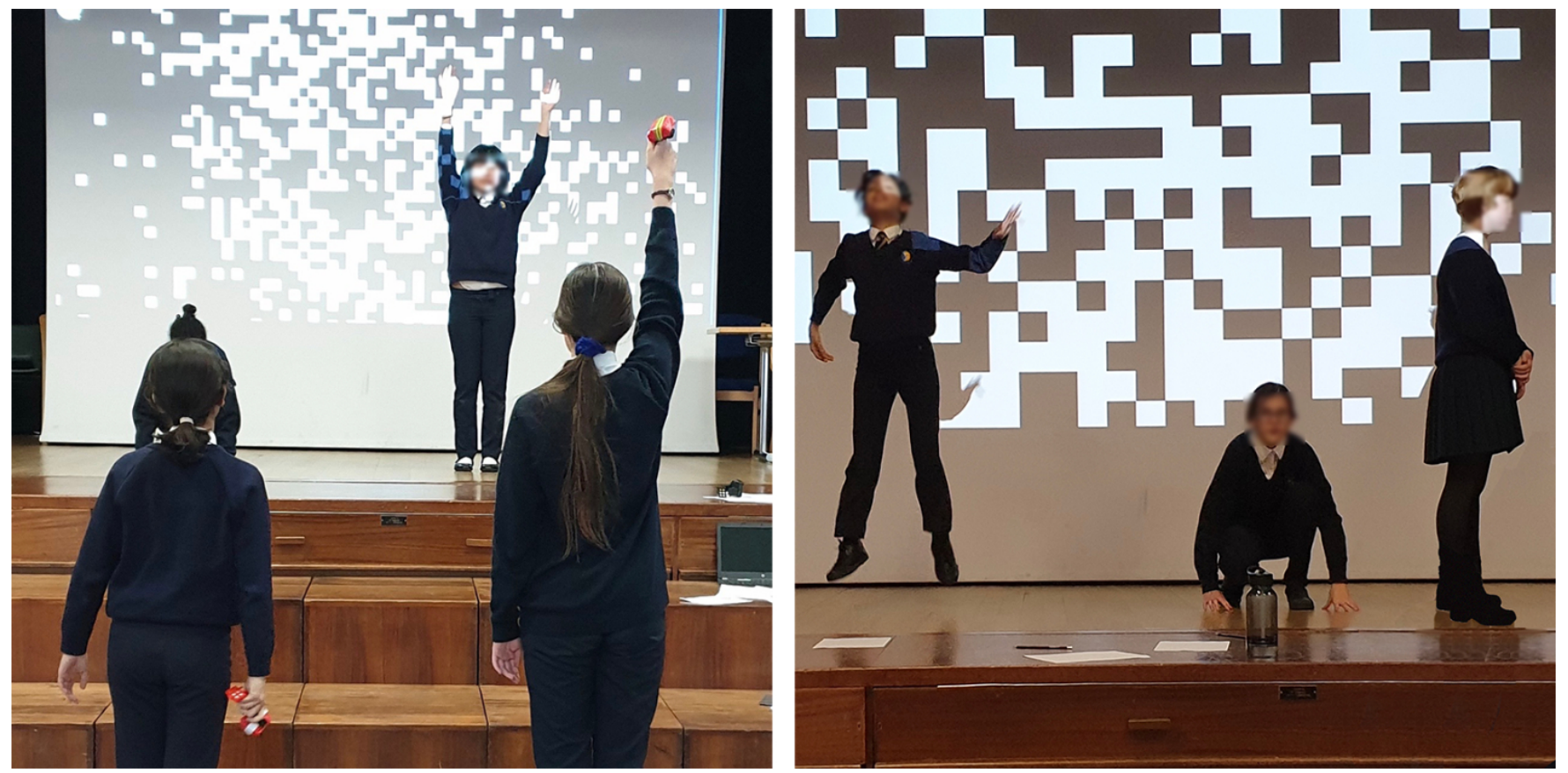

Figure 1: Students moving and interacting with the DataMoves devices.

Permission to make digital or hard copies of all or part of this work for personal or classroom use is granted without fee provided that copies are not made or distributed for profit or commercial advantage and that copies bear this notice and the full citation on the first page. Copyrights for components of this work owned by others than the author(s) must be honored. Abstracting with credit is permitted. To copy otherwise, or republish, to post on servers or to redistribute to lists, requires prior specific permission and/or a fee. Request permissions from permissions@acm.org.

DIS '21, fune 28-fuly 2, 2021, Virtual Event, USA

(C) 2021 Copyright held by the owner/author(s). Publication rights licensed to ACM. ACM ISBN 978-1-4503-8476-6/21/06 . \$ \$15.00

https://doi.org/10.1145/3461778.3462039

\section{ABSTRACT}

In the domain of computing education for children, much work has been done to devise creative and engaging methods of teaching about programming. However, there are many other fundamental aspects of computing that have so far received relatively less attention. This work explores how the topics of number systems and data representation can be taught in a way that piques curiosity and captures learners' imaginations. Specifically, we present the design of two interactive physical computing artefacts, which we 
collectively call DataMoves, that enable students, 12-14 years old, to explore number systems and data through embodied movement and dance. Our evaluation of DataMoves, used in tandem with other pedagogical methods, demonstrates that the form of embodied, exploration-based learning adopted has much potential for deepening students' understandings of computing topics, as well as for shaping positive perceptions of topics that are traditionally considered boring and dull.

\section{CCS CONCEPTS}

- Human-centered computing $\rightarrow$ Human computer interaction (HCI); • Social and professional topics $\rightarrow$ Computing education; • Applied computing $\rightarrow$ Interactive learning environments.

\section{KEYWORDS}

Education/Learning, Embodied Interaction, Schools/Educational Setting

\section{ACM Reference Format:}

Justas Brazauskas, Susan Lechelt, Ethan Wood, Rebecca Evans, Su Adams, Emma McFarland, Nicolai Marquardt, and Yvonne Rogers. 2021. DataMoves: Entangling Data and Movement to Support Computer Science Education. In Designing Interactive Systems Conference 2021 (DIS '21), fune 28-fuly 2, 2021, Virtual Event, USA. ACM, New York, NY, USA, 15 pages. https: //doi.org/10.1145/3461778.3462039

\section{INTRODUCTION}

The widespread push to popularise computer science education has largely focused on digital literacy, computational thinking and problem solving [16], where the latter two are often used as a way of introducing programming. The overarching goal is to teach children to code. While learning to code is without question a fundamental digital literacy skill all children should learn, too much emphasis on coding may result in other computer science topics, such as abstract computational concepts (e.g., binary, logic) being short-changed in the curriculum and covered in a more cursory way, which potentially could deter children from becoming more interested in them [43]. Furthermore, the way such topics have been traditionally taught in classrooms is often perceived as dull and boring [3]. How might these other aspects of computer science be introduced to learners in a more engaging and thought-provoking way, that could lead to them developing a positive attitude towards the subject and a deeper understanding of it?

One approach to revitalise computing classes has been to introduce interdisciplinary teaching, where different perspectives are used to introduce computing topics [21]. This has been explored for a number of domains, for example when teaching about history together with digital fabrication [10], using computationallyaugmented sewing and craft activities to broaden participation in computing [23], and asking learners to produce technologies to support their dance practice [13]. These forms of interdisciplinary teaching can result in more engaging and relevant ways of learning. Another approach is to use physical computing devices to broaden the scope of teaching computer science concepts, such as sensing and IoT $[12,24]$ - for example, through the use of toolkits like the micro:bit, Raspberry Pi, and Arduino [6, 11, 39]. However, the focus is still on learning to write software, but with the added activities of tinkering and problem solving.

Here, we propose a different, more embodied approach for teaching computer science concepts: one that emphasizes the entanglements of movement and data, where one flows into the other when in a state of flux, and where data and movement relate to one another. To achieve this more fluid and dynamic style of learning experience, we designed and experimented with a diversity of physical computing devices that could be used to create and control a range of software visualisations, for the abstract concepts of data representation and number systems. Our rationale for reconceptualizing these concepts in this manner, i.e., as 'data moves', was to provide students with a new way of understanding, transposing and visualising number systems and data - for example, to experience the magic in changing a Roman number into ASCII and then into decimal through a software visualisation. Teachers have often commented on how difficult it is to get children excited by these topics; they are often perceived to be dull. Our challenge was to transform this preconception.

The goal of our research, therefore, was to see how we could make number systems and data representation come alive. We approached this by enabling them to be linked through technology and experienced by groups of 12- to 14-year-old students through physical movement and embodied exploration. A choreographer and educational instructor joined us in our design research, through participating in a series of collaborative design sessions for this purpose, where together we envisioned and contested how to mould and meld novel technologies into their teaching practices. The outcome was the creation of a set of physical computing devices, together with a diversity of learning activities - sometimes supported by the technology and sometimes without - that could enable the topics of number systems and data representation to be explored from multiple perspectives over a period of time; including through hands-on activities, problem-solving, group creativity, traditional paper-based instruction and physical and mental exercises.

The main contribution of our research is to demonstrate how it is possible for children to experience computer science concepts in more engaging and embodied ways, through using physical computing devices as a resource that taps into teachers' and children's creativity and imagination and which can provide deeper understandings of them than is taught in traditional classroom settings. Next, we cover the background in embodied learning and tools (Section 2), then proceed to discuss the conception and the design of the DataMoves devices (Section 3 and Section 4). We then present the workshops that were run and what happened during them (Section 5), followed by our evaluation (Section 6). Finally, we provide an in-depth discussion and future work (Section 7) and conclusions (Section 8).

\section{RELATED WORK}

The literature reviewed here covers research on embodied learning, how physical computing tools have been developed and used in teaching children coding and computing, and the role of materials for designing new learning interactions. 


\subsection{Merging embodied learning and physical computing}

In recent years, there has been an increase in research exploring how embodied interaction, coupled with physical artefacts can support learning. By embodiment is meant the connection between human reasoning and our bodies, and their physical/social experience in the world [30]. Embodied interaction can leverage children's pre-existing knowledge of the physical and social worlds to facilitate understanding when learning with artefacts (e.g. [14, 22, 29]) Moreover, it has been suggested that it can support the offloading of cognition into the real world, and in doing so link abstract ideas to external representations [5], which can be leveraged for teaching about abstract topics like data science and computing [33].

A number of studies have pointed to the positive linkages between STEM learning and embodied movement [1] - for example, exploring how to couple gestures signifying mathematical operations [40] and geometric properties [7]. Researchers have also explored how an embodied, lived experience with physical artefacts can engage children in reflecting about data and data literacy concepts (e.g., [4, 8, 39]. The pioneering Ambient Wood project [35], for example, developed technologies to enable children to broaden and connect their understandings, reflections, and hypotheses about data, through using a variety of mobile and standalone computational devices to explore an outdoor environment. Visualisation tools were also provided that enabled access to and the sharing of contextually relevant digital information. Groups of children were able to develop a deep understanding of the environment, based initially on data they had collected about it using their own sensing and probing devices, and then subsequently based on their reflections of the data in the form of abstracted patterns appearing in an overall data visualisation. Being able to see their own and others' data in this highly engaging way, enabled the children to make more connections, and to learn how to abstract and generalize from it. In a similar vein, capitalizing on a lived experience, Lee et al. [28] engaged children in movement-based activities with their personal tracking devices. Specifically, children were given Fitbit devices to track their movement, and then used the data collected to compare how many steps they had taken in relation to others. The process of examining and discussing collected data about their bodies and movement, supported the children's understanding of statistical concepts like mean and median, consolidating the relationship between understanding abstract ideas and their physical exemplification.

More recently, others have investigated how physical and mobile computing devices can be coupled with exploration-based, embodied learning to teach about other abstract computing topics, including machine learning and Internet of Things (IoT). Hitron et al. created a physical computing tool called the "Node" $[18,19]$ to support children in learning about machine learning concepts. Using the tool, children experimented with data labelling and evaluation aspects of machine learning, through acting out various physical gestures, which the system then attempted to recognize and classify. Experimenting with the system in this way helped the children to learn about what happens behind typical "black-box" machine learning algorithms. The "Node" was further repurposed as a tool for fostering outdoor play for children, that could be used in combination with a coding platform that controlled the device [31]. Children could either create new games or modify existing ones by writing code and experimenting with the system's physical hardware. In this way, the tool was able to augment the experience of outdoor play, while improving their understanding of computing overall.

To foster playful, exploration-based learning about IoT concepts, Lechelt et al. developed the Magic Cubes which were handheld, physical computing tools made from modified Arduino hardware with embedded sensors [25, 36]. Their emphasis was to encourage the development of curiosity and critical thinking about IoT sensors and networked systems, without necessitating children to program IoT hardware. Instead, the children used the Magic Cubes to move around the classroom, sensing aspects of their bodies and the environment in order to explore how reliable, accurate and informative data was in different contexts. This embodied approach was demonstrated to enable the children to engage in scientific enquiry and critical thinking, as well as to understand and articulate IoT concepts, including data collection, noisy data and the (un)reliability of data.

\subsection{Materials for learning}

Much research to date has used novel physical computing devices either as tools to sense and explore aspects of the environment or human body from which to make abstractions (e.g. [24, 28, 35]), or as a platform to program on (e.g. [20, 27, 39]). An alternative approach is to conceive of physical computing technologies more broadly, as possible materials to be experimented with alongside other paper-based materials and artefacts already used by teachers when developing new activities. In this sense, they are not foregrounded to fulfil a particular goal, but are viewed as one of a range of materials and tools that are available to the instructors to use in a methodological bricolage-like manner (cf. [34, 41, 42]).

Conceptualising the technology in this way, enables designers to have more scope to experiment with hardware and software, especially when thinking about how to integrate them into learning activities. Having a looser coupling and framing between the tools available and the learning, means they can also explore when and where it might be suitable to use them in conjunction with other paper-based and activity-based methods. A benefit of this approach is that it can enable both teachers and children to explore how to understand abstract concepts through experimenting, creating, recalling, observing and generating sequences and patterns (cf. [32]). Our design research approach was also inspired by Rosner's [38] ideas about the potential of using alternative materials for interaction; where different tangible, tactile and embodied skills are activated in the encounter with different materials evoking different ideas, memories and forms of reflection and engagement - be they paper cards, physical computing devices, data visualisations and so on. Here, our focus is a learning context rather than a design context; where the materials are chosen to match and build upon knowledge that the learner is encountering and experiencing through hands on activities and body movements [15].

\section{COLLABORATIVE DESIGN RESEARCH}

We adopted a design-oriented research approach, where we explored how to develop new physical computing devices that could 
be used and combined with other materials and forms of learning activities, to extend the range of learning interactions through which children can learn about computer science. Our goal was to develop and deploy a one-day workshop for students aged 12-14 years, aimed at teaching them about data representation and number systems in an embodied, engaging, and creative way. We wanted to support the students' learning with bespoke physical computing devices, which would enable them to embed their understanding of the chosen computing topics through movement and choreography. Throughout the design process, we worked as an interdisciplinary team, comprising: academic staff whose role it was to build the physical devices and conduct research in the wild using them (A), a computing curriculum specialist (C), and a dance choreographer (D). C had a background in training teachers in computer science education and her role was to lead co-development of the learning materials; whereas D had a background in educating a range of people in STEAM subjects through dance, and her role was to lead the co-development of movement-based learning activities to be used alongside the physical computing devices. The design research process took place through a series of collaborative sessions, where we: (1) ideated the pedagogical goals for the workshop and the design criteria for the physical computing devices; (2) designed the devices; and (3) developed the workshop structure. Finally, it culminated in the deployment of the designed workshop, together with the developed devices, in two different school settings.

\subsection{Deciding on the pedagogical goals of the workshop}

As a first step, our interdisciplinary team met to discuss the scope of the envisioned workshop and its pedagogical goals. Together, we agreed to develop a one-day reusable workshop, for which we would design bespoke physical computing devices, and that could be integrated into D and C's broader programme of dance and computer science workshops that they had already successfully set up for running in a diversity of UK schools. A fundamental principle behind D and C's approach to designing educational workshops is to promote the use of activities, technology, and other everyday materials that "inject creativity and energy" into learning. Their unique approach is to combine dancing and choreography with computing, including experimenting with different technologies in the choreographic process, and understanding the organisational and choreographic needs of working with digital concepts.

For the new workshop, we chose to develop an immersive digital dance experience for 12-14-year-olds that would enable them to explore, grasp and apply the topics of number systems and data representation through a variety of both group-based and individual learning activities. Specifically, we chose to explore how to teach a number of concepts under the umbrella of number systems and data representation, which were: binary, ASCII character encoding, pixels and resolution. The reason for this is that these concepts mapped directly to the English computing curriculum for this age group, and we considered them fundamental to understanding the broader chosen topics. Part of the workshop would involve experimenting with data representations and visualisations using the physical computing devices, alongside learning through other low-tech materials and physical artefacts.
The workshops that $\mathrm{D}$ and $\mathrm{C}$ had been previously developed, had so far focused largely on teaching core programming concepts. During their workshops D and C would take alternating roles, where $\mathrm{C}$ would explain theoretical concepts to students, followed by D's creative interpretation that would make the information more accessible by engaging the students with it in a different, embodied way. For the DataMoves project, they were interested in exploring more how movement and digital tools could be used to teach and embed the learning of other aspects of the computing curriculum. This was going to be the first time they applied the method to learning about data representation number systems. It is often thought that the topics of number systems and data representation are dry to teach, with many students disengaging and not understanding the relevance of the subjects to them and in turn not embedding the knowledge around these subjects. Our aim, therefore, was to design new physical computing devices that could be combined with movement to teach these concepts in new ways, providing clear connections and benefitting different types of learner, whilst also allowing us to explore whether this enabled a deeper understanding and embedding of the learning.

In our design process, we also chose to adopt a form of ludic engineering where we promote learning through novel, playful visions of technology $[9,37]$. We look at the technologies themselves as a source of inspiration to see what they might suggest to us, feeling free to configure and reconfigure them for emergent ideas. Having technologists, designers, artists, researchers, doers, and others, who typically would not normally work together, combine their skills and ideas enables us to promote new views on what is possible. We also acknowledged that the physical computing technology should not dominate the workshop but be used judiciously when thinking about how to augment the learning. Adopting such an open-ended approach meant that the devices we built could be used when deemed appropriate for a given learning activity and even be used more generally to play with at certain moments for inspiration and creativity.

\subsection{Formulating the core design criteria for the DataMoves devices}

Having decided on the workshop goals, we next discussed the design criteria for the physical computing devices to be designed for the workshop - which we call the DataMoves devices. While we wanted to design the DataMoves devices to be open-ended to encourage children to explore computing concepts from a creative perspective, we were mindful that they would need to be robust enough to be used by groups of energetic children, easy to deploy in very different school/community settings and be flexible enough for adapting to a range of collaborative and individual activities. To help in the initial stages of ideation, through discussion, we came up with a set of three design criteria. As well as the devices needing to be robust, it was considered key that they:

(1) Enable group and individual interactions.

(2) Enhance learning about computing concepts through discovery and experimentation.

(3) Afford creativity in how they can be used/adapted. 
It was also considered important to provide a means to teach a relevant element from the English computing curriculum programme, in a way that enhanced the learning and benefitted the students.

\section{Enable group and individual interactions}

The team decided it was important that the DataMoves devices enable both group-based and individual interactions. The reason for including group-based learning, in addition to dance being a social and collaborative activity, is that group-based learning is known to facilitate critical thinking skills, evaluation of others' ideas and learning of factual knowledge [17]. We thought about how we could afford group-based learning, through designing for multi-user interactions within a single device. However, early in the design process we noticed that not all of our ideas for data visualisation and interaction were compatible with being used by several users at the same time. We thus, chose to design two kinds of devices that could be alternated between: those that could be used individually, and those that supported multi-user interaction.

\section{Enhance learning through discovery and experimentation}

A key goal was to enable the children to explore computing concepts without necessitating coding - and instead, learn about them through discovery and exploration. This goal was chosen in line with other research on discovery-based learning, which has demonstrated how it can be an effective and engaging means to introducing learners to complex concepts that are new to them $[19,24]$. Another requirement was to determine how to fill a whole day workshop to ensure that learning deepens over time and builds upon previous activities, while ensuring that the children don't get bored. This challenge is quite different from devising, for example, a short classroom lesson and requires thinking about how the devices can be used for multiple purposes and interactions. Our idea was to create devices that would enable a series of exploration-based activities that could build upon each other and could be combined with movement and choreography in order to understand the computer science topics being taught and be able to apply them in practice.

\section{Afford creativity in how they can be used/adapted}

Given D and C's focus on enabling creativity as a means for making computing education exciting and engaging, we decided a key requirement for the devices to be that they afford creativity in how they can be used/adapted by the students. Specifically, we wanted to ensure the interaction afforded by the designed devices was not always prescriptive, and that the devices could be appropriated by the students in creative ways that were not pre-defined by us. One of the ideas we had to achieve this, was to create a range of software tools to go together with the hardware design, that would allow students to select different visualisations which could be used either as the focal point or supplementary background material. We also considered how designing devices that would be capable of being used on their own, or in conjunction with other tools, e.g., pen and paper, might afford more creative appropriation and adaptation of the devices.

\section{DESIGNING THE DATAMOVES DEVICES}

Next, we held three sessions with the whole team to ideate and discuss alternative designs. In these meetings, $C$ first suggested and presented the concepts to be taught during the workshop (e.g., binary, ASCII, resolution), discussing what children need to know about them, based on the computing curriculum. Binary and ASCII were selected as especially important concepts, as they illustrate how computers use different data representation models beyond what we are used to e.g. base 10 notation, where each digit in a position of a number can have a value of 0 to 9 . D then led ideation of sample movements and choreographies that could be used to teach the topics - for example how standing up and crouching down might represent "embodying" a binary number of 1 or 0 . Finally, A commented on the technical feasibility of registering these movements using sensors and suggested how physical computing might be used to support the curricular content. The process was iterative; throughout the sessions, we came up with a large range of ideas, then narrowed them down and iterated further on the ones that best matched our agreed pedagogical goals and design criteria.

Prototyping some of our initial ideas helped us realize how well they could be expected to work in practice. For example, in the first session, one of the initial ideas we had was to design an Arduinobased Geiger counter to allow the children to explore the radioactive properties of artefacts through sensing and representing the data using audio and visual representations. The device was conceived as a way of sensing invisible particles to teach students about both data representation and physics. However, after some initial prototyping and testing of a Geiger counter we found that despite its evocative qualities for provoking exploration of data collection, it was limited in what else it could be used for, what it could detect and how quickly it could do this.

As a result of this collaborative ideation and prototyping process, we ultimately decided to develop two different, flexible devices that could be mapped onto the target learning concepts, and used to explore number systems, data, choreography, and movement in a variety of ways. These were (a) a tethered device in the form of a physical, interactive base station (b) a wearable device with the form factor of a wristwatch (see Figure 2). The base station was designed to be used collaboratively, whereas the wearable was intended to be worn by one person at a time.

We then developed the hardware for the two devices and created corresponding software that could provide a range of visualisations for creatively displaying the sensor-based output from the devices on a connected screen (i.e., a projector). The software was designed so that it could be easily adapted, and where other visualisations could be added to the set later. This enabled feedback from D and C to be elicited when they started to envision how the hardware and software combined could be experimented with in their proposed learning activities. For both the wearable and the base station, we designed both "open-ended" visualisations and more "concrete" visualisations using the new devices, which are explained in more detail in the next subsections. The aim of the open-ended visualisations was to be exploratory, discovery-based and to encourage experimentation. They were primarily intended to be used as an introduction to each device, in order to enable students to become comfortable using them. In contrast, the concrete visualisations 
were aimed more specifically to convey a learning concept related to number systems and data representation. A menu of the different types of visualisations was created for both devices to allow users to be able to choose and explore different mappings.

After creating detailed sketches, we built the two devices using generic off-the-shelf electronic components, and 3D printed bespoke plastic casing. We programmed the devices using Arduino IDE and wrote software for them using Processing. Both devices wirelessly connect to a host computer that was running the visualisation script that enabled the users to see how their actions mapped to the visual output. Next, we describe each of the two devices in more detail, along with their design rationale.

\subsection{Base Station}

The base station is an octagon-shaped device with a total of 8 distance sensors, one on each side (see Figure 3). Each of the distance sensors measures how far an object is away from it, which the software then visualises on a screen. Students can interact with the base station by triggering the sensors through changing their position in space, in relation to the distance sensor, and by doing so changing the output on screen. The shape of the base station was conceived as a way of enabling exploration of the ASCII number system, which is based on eight binary bits (0s and 1s) - with each sensor serving as an input device for a binary bit. It was also designed to focus on tracking lower body movements, where groups can change their body position to trigger the sensors which then visualise and map their location in space on a shared public display.
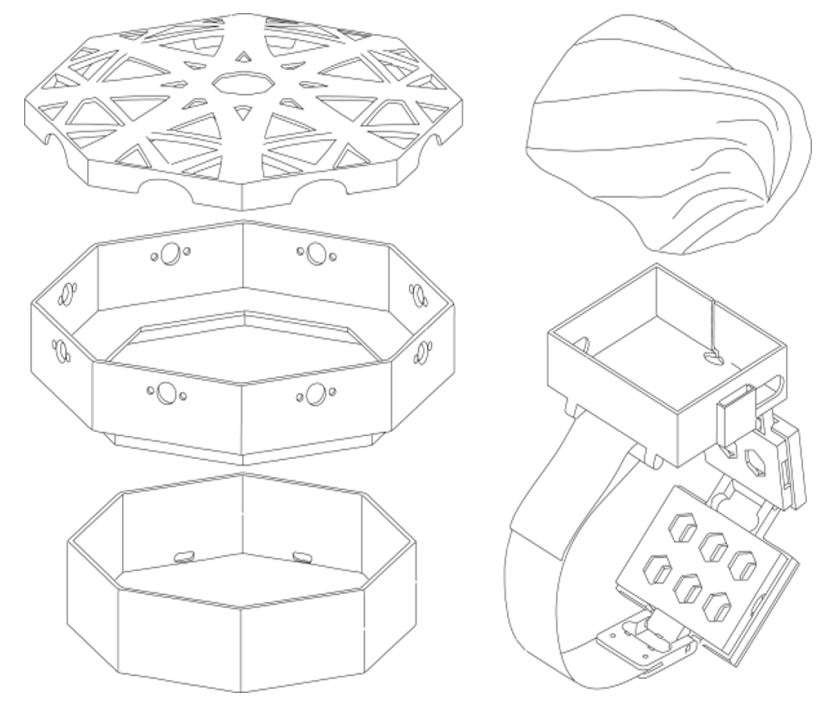

Figure 2: Sketches of the base station (left) and the wearable device (right).

The design idea behind the base station was that it could be positioned in the middle of a space where up to 8 individuals could stand - one in front of each sensor - close to a projected display that would show the sensors reacting to their physical movements in real time. The shape of the device was also designed to afford how to use it: the octagon intrinsically indicates 8 directions and is aimed at subtly guiding the students where to stand. To achieve long-range, low-latency proximity sensing, we used Time-of-flight (VL53L1X) sensors, making the base station have a sensing radius of 4 meters in each of the 8 directions. To be able to connect 8 I2C sensors to an Arduino Nano 33 IoT we used an I2C multiplexer, along with a HC-06 Bluetooth module.

The corresponding software was designed to visualise, in real time, the students' bodies moving around and perpendicular to the device, while also representing concepts related to binary. The visualisations used the sensor data in both continuous and discrete modes. In the continuous mode, the students could change the shape and size of the abstract geometry, that they could observe on the screen, for example by moving closer to or further away from the device (see Figure 4A, Figure 4C). This was aimed at helping the students familiarise themselves with the device while also enabling them to explore the boundaries of what the station could sense. These kinds of 'fluid' visualisations were designed to be open-ended and exploratory. In contrast, the visualisations created for the discrete data mode were designed to be more concrete and intended to convey specific learning concepts. In particular, the distance threshold was set on each sensor output to be either 0 to 1 ; this had the effect of the visualisation being on or off on the screen (see Figure 4B, Figure 4D). These 'discrete' visualisations were used to represent specific aspects of number systems, for example enabling each student to "become" a bit by representing either a 1 or 0 based on their position in space. They were also designed to enable the users to work together to create meaningful multi-bit sequences, for example to create together an 8-bit ASCII character.
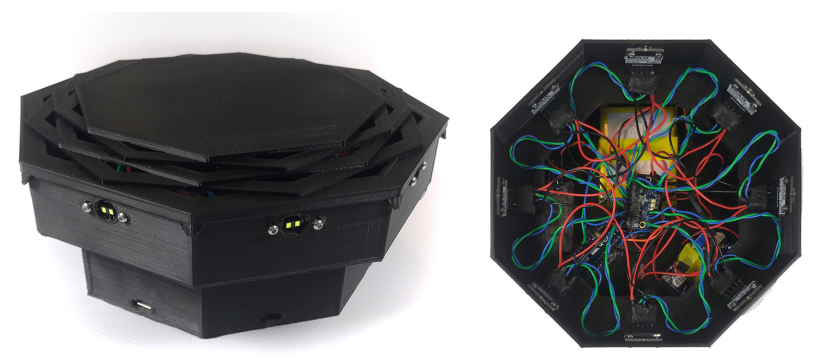

Figure 3: The inside and outside of the base station showing wiring and movement detection sensors on each side.

\subsection{The Wearable}

The wearable device (see Figure 5) was designed with a 6 axis Inertial Measurement Unit (IMU) embedded in it, enabling the wearer to explore a range of digital representations in front of a wirelessly connected display (e.g., a projector screen). It provides real time feedback in the form of dynamic visualisations that the user "paints" on a connected display, as a result of moving the limb that has the wearable attached. The device was designed to be placed on the wrist and to be actuated through arm movements, making it easy for the wearer to experiment with and explore the data visualisations. It was also designed to be rugged, to minimise the risk of potential harm to the wearers, e.g., scratches, induced by careless moves. The user secures the device on their limb, using a Velcro 


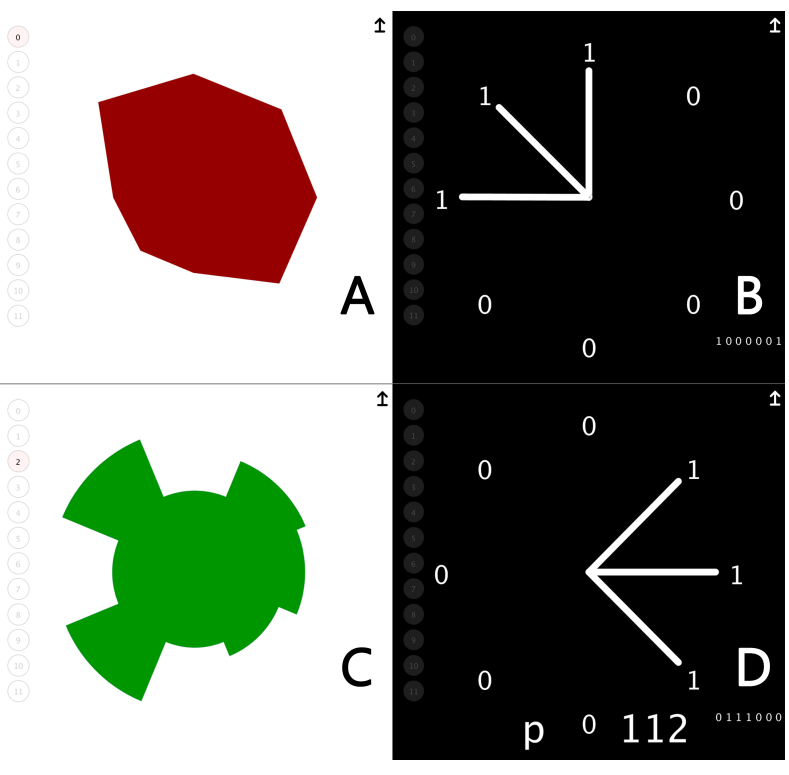

Figure 4: Screenshots of some of the visualisations developed for use when moving in front of the sensors embedded in the base station. (A) and (C) are examples of "open-ended" visualisations while (B) and (D) are examples of "concrete" visualisations; (B) is a Binary visualisation using lines that denotes 0s and 1s, while (D) is an ASCII visualisation that points to $1 \mathrm{~s}$ and 0 s and shows the number underneath.

strap that is connected to a 3D-printed wristband, which houses the electronic components.

The software was designed to provide a mapping between the sensor readings and the computing concepts of pixels and resolution through the displayed visualisations. All of the visualisations directly mapped the IMU readings to the "painted" location on the screen. Specifically, they were designed to enable the students to "draw" on the screen using the direction of movement and inertia of one of their limbs. The visualisations were designed using different resolutions of pixels and colors (see Figure 6 and Figure 7). They were designed to provide a range of visualisation tools that the teachers and students could experiment with. The settings to do this were easy to control while wearing the device - akin to having a palette at hand. The visualisations were all designed to both be able to be used in an open-ended and experimental way, and more concretely as a way to support learning about specific concepts. For example, the concept of resolution was represented by the visualisations shown in Figure 7 (A) and (B), where moving one's limb to visualisation setting (A) produced an image in a low resolution and moving it to visualisation setting (B) produced an image in a high resolution. Furthermore, the resolution visualisations (Figure 7) were designed to also help understand the binary number system as each square was either on or off (which was shown as squares either being white or black).

To support learning about color depth, moving one's limb to a 2-bit color visualisation produced patchy and discrete color lines, whereas moving it to an 8-bit color visualisation produced much smoother colors (see Figure 6). This was aimed to demonstrate, in an embodied way, how using more bits could lead to an increased range of outputs. In sum, the visualisations created for the wearable were designed with increasingly finer granularity transitions, to illustrate how a higher bit representation correlates with more information being displayed.

\section{DESIGNING THE WORKSHOPS}

Having designed and developed the two DataMoves devices, we held one further collaborative ideation session, where we experimented with them as a team to decide specifically how they could be used to support learning about number systems and data representation. A further goal was to conceptualize and develop the envisaged full-day workshop, which would include a range of learning activities, some of which would call upon the DataMoves devices, and some of which would not. D and $\mathrm{C}$ proposed that in addition to using the DataMoves devices, the workshop should also use other kinds of physical objects as well to support movement-based activities. The use of pen and paper was also incorporated into the learning activities for this purpose. These were intended to allow the students to practice what they had learned in a more traditional way. This format of using novel technologies followed by traditional materials, and vice versa, was intended to support the students in switching perspectives when learning and practicing the content through a range of learning modes. The importance of using movement and embodied interaction to explore the concepts was considered when developing the entire workshop structure.

The key concepts that we decided to cover under the topics of number systems and data representation were: binary, denary, ASCII character encoding, pixels, resolution and RGB color depth. The reason for this was that we envisioned these concepts as complementing each other, and as collectively having the potential to provide a deep understanding of the broader topics of number systems and data representation. During the ideation session, we discussed how to use the DataMoves devices for the workshop, and how to adapt D and C's existing choreography and group movement activities from their other workshops, to map onto the new topics of data representation and number systems. For example, we discussed: how to introduce the DataMoves tools through exploration; how movement-based improvisation tasks might convey binary place value headings; and creative tasks that led students through choreographing their own movement sequence to reinforce their understandings of number systems. The DataMoves devices were appropriated in the workshop so as to be used intermittently throughout it rather than wholly driving it. This included at the beginning, after sections of the workshop on binary numbers and ASCII to help consolidate learning, and several times throughout the remainder of the session to demonstrate the concepts of pixels and resolution. This was considered important, so that the children did not fixate on the technology but used it to explore the concepts they were learning, discovering and choreographing movements for.

We decided that binary bits should be introduced first because they are a fundamental basis to the other concepts including ASCII and resolution. We considered that getting the students to embody bits, by enacting $1 \mathrm{~s}$ and 0 s using the base station, as a first learning 

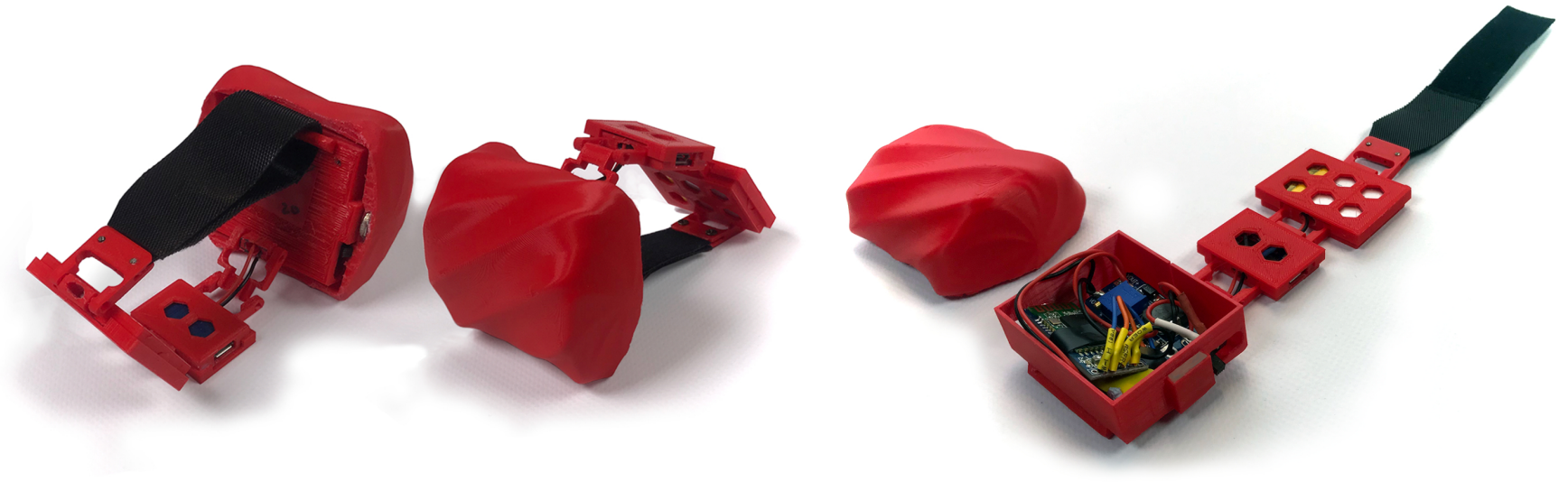

Figure 5: The inside and outside of the wearable showing wiring and casing.

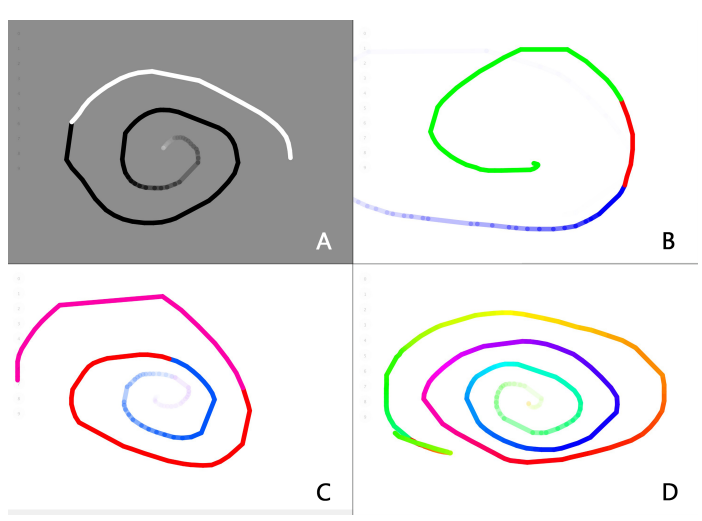

Figure 6: Examples of the wearable color depth visualisations provided: (A) a 1-bit color line in black/white, (B) a 2-bit color line (C) a 4-bit color line, (D)an 8-bit color line.

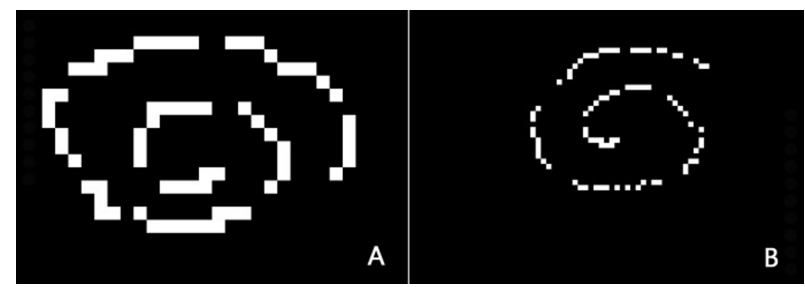

Figure 7: (A) Low and (B) high pixel resolution visualisation examples.

activity could be easy, engaging and get them comfortable with the basics of the binary number system. We then decided to use technology-free activities get the students to explore how bits are combined into bytes, and how these can be translated into other representation systems, like denary (i.e., integers). The reason for not using the devices at this stage, is because this is something that the students would have learned previously in class; we wanted them to remember the pen and paper process of reading and translating binary they had already learned, and then help them consolidate this knowledge in a different way, using embodied movement. It was decided that ASCII should be introduced next, as this could be done using the base station on a different visualisation setting in groups, helping the students directly build up their knowledge further, while using the same device.

The concepts of pixels and resolution were decided to be taught together, using the wearables. Since there were parallels between these concepts and those related to binary, i.e., pixels having two states (on/off) mirroring that of binary data, we decided to highlight these parallels when verbally explaining them to the students, then to let them explore the visualisations on the wearables. To introduce color depth, we decided to verbally introduce the combined theory behind bits, pixels and resolution to show how with an increasing number of bits, we can gradually represent richer data. Finally, the students were then invited to view and demonstrate the different visualisations comparing how colors are represented in different depths from 1 bit ( 2 colors) to 8bit ( 256 colors).

Next, we describe the detailed structure of the final workshop that we agreed on along with its rationale, highlighting how and when the DataMoves devices played a role.

\subsection{Final Workshop Structure}

Figure 8 shows the finalized structure of the workshop, with planned activities, timings, tools and illustrations of each of the tools. The same workshop was run in two different schools, which we describe in detail in Section 6. To begin, the workshop started with a movement-based warm-up activity. This served as an icebreaker introducing the students to the research team and to what was in store for them in the workshop. Next, the students were asked to get into groups of four. They then spent 20 minutes engaging in open-ended exploration with the base station and the wearables, which was aimed to get them comfortable with using the two devices and getting an initial sense of how they work. The devices were distributed throughout the physical space of the room where the workshop was being held. The students were told that they could switch between them and experiment with the different visualisations. 
Following these introductory tasks, the next 2.5 hours of the workshop were subdivided into three separate but interrelated sections about the number and data concepts: 1 ) binary and denary number systems, 2) ASCII character encoding and 3) pixels and resolution. For each, the key concepts were verbally explained, and the interactive tools, along with other forms of activities, were used to help the students consolidate their understanding of the concepts. Each section included both group-based and individual learning activities, as well as structured and creative components; the structured components aimed to convey and teach a concept, and the creative components aimed to enable the students to apply that concept imaginatively.

In the first section on binary and denary number systems, the students were initially asked to experiment with the base station to see how their movement in relation to the distance sensors (e.g., stepping towards and further away from the sensor) caused the connected visualisations to output either 0 s or 1 s (see Figure $4 \mathrm{~B})$. This enabled them to explore how binary numbers relate to discrete, rather than continuous, states. Experiencing how a state can change through using their physical presence in the space to generate $1 \mathrm{~s}$ and $0 \mathrm{~s}$ on the base station, was then used to scaffold a group discussion about the concept of switches in a computer and the relationship between the number 1 meaning "on" and 0 meaning "off".

Next, a group discussion took place about how to combine bits together to create bytes, and to learn how to understand how to read binary 4-bit numbers. They were then asked to translate these to denary (integer) counterparts. A movement-based task (that did not include the DataMoves devices) then took place where the groups of 4 students were asked to create their own dance to represent a 4-bit number. For this, they were tasked to enact out bits - devising one movement to mean "on" and another to mean "off" and then performing the movements together to form a 4-bit binary number. Each group performed their 'bit dance' to the other groups. The other groups were then asked to translate the 4-bit number to a denary integer (for example with the binary number "0101" translating to the denary integer " 5 "). In this way, the students were able to explore how data moves through different number systems by abstracting and acting out sequences.

The second section covered ASCII character encoding. The students worked this time in larger groups of 8 to be able to work on larger number patterns. In particular, they were asked to first choose an ASCII character and then look up its 8-bit representation with the help of a paper chart. They were then given the challenge of using the base station collaboratively to "encode" the ASCII character, with each student deciding to physically represent either a 1 or a 0 , while the students in the other groups watched and tried to decode the character they were representing (see Figure 4D). This element of fun and challenge was aimed at making the activity of encoding and decoding the ASCII numbers engaging.

The last section combined learning about pixels, resolution and RGB color depth. For this, the wearables were used. Rather than asking all of the students to experiment with the wearables, it was decided to use them as a way of demonstrating the concepts, where one or two students would be called to the front of the workshop studio to try them out. One of the instructors (C) first talked through the concepts of pixels, resolution and color depth.
Next, the volunteer was asked to dance and move while wearing the wearable on their arm, as the instructor changed the sensorbased visualisations that were displayed on the projector screen. In this way, the students were able to experience how dancing along with a lower versus higher resolution produced images of different levels of pixelation on the public display while the other students were watching. A similar demonstration was performed to scaffold discussion about color depth (see Figure 6 and Figure 7 for examples of how the visualisations changed based on resolution).

The final group activity in the workshop was an open-ended and creative one, where groups of 4 students were asked to use any of the paper-based tools used throughout the workshop or the DataMoves devices to demonstrate their understanding of the concepts they had been experiencing and learning about. They were given 45 minutes to come up with a choreography, which would demonstrate their understanding of two of the concepts of their choosing that were covered during the day, which they then performed to the rest of the class. After performing their choreography, they were asked to explain to the rest of the groups how it related to the topics that had been covered throughout the day - i.e., number systems, ASCII, pixels, or resolution.

\section{WORKSHOP DEPLOYMENT}

We ran two full-day workshops in two city-based, mainstream schools in London, England. Each workshop was attended by 16 students who were 12 to 14 years old. Both took place during a school day, over the course of 4 hours, excluding breaks. The students were chosen to take part in the workshops by their teachers. Workshop A was held in the auditorium of an all-girls school, while Workshop B was held in the dance room of a mixed gender school that specialized in the performing arts. According to their teachers, the students in both schools had had a rudimentary introduction to number systems and data representation topics earlier in the school year, but the workshop as a whole went beyond what the students had been exposed to in terms of these topics. The workshops were led by the computing curriculum specialist (C) and the dance choreographer (D). In addition, two academic researchers were present at each workshop, to observe the workshops taking place and to collect data.

The workshops were evaluated, using a qualitative approach, to determine how well the devices performed in terms of our design criteria. Specifically, we wanted to understand (i) to what extent the DataMoves devices enabled collaborative learning, (ii) whether the exploration and discovery they afforded supported the students' learning, and (iii) to what extent the DataMoves devices afforded creativity. Another goal was to (iv) understand the students' perspectives on the workshop, and how they felt it compared to a typical computing class. To answer these questions, we collected: (1) observation notes during the workshops and (2) interviews with a subset of the students and teachers after the workshops. In our observations and subsequent discussions, we did not find marked differences between how the students' interacted in the two different school settings. Therefore, we present our combined analysis from both workshops. Next, we describe the data collection and 


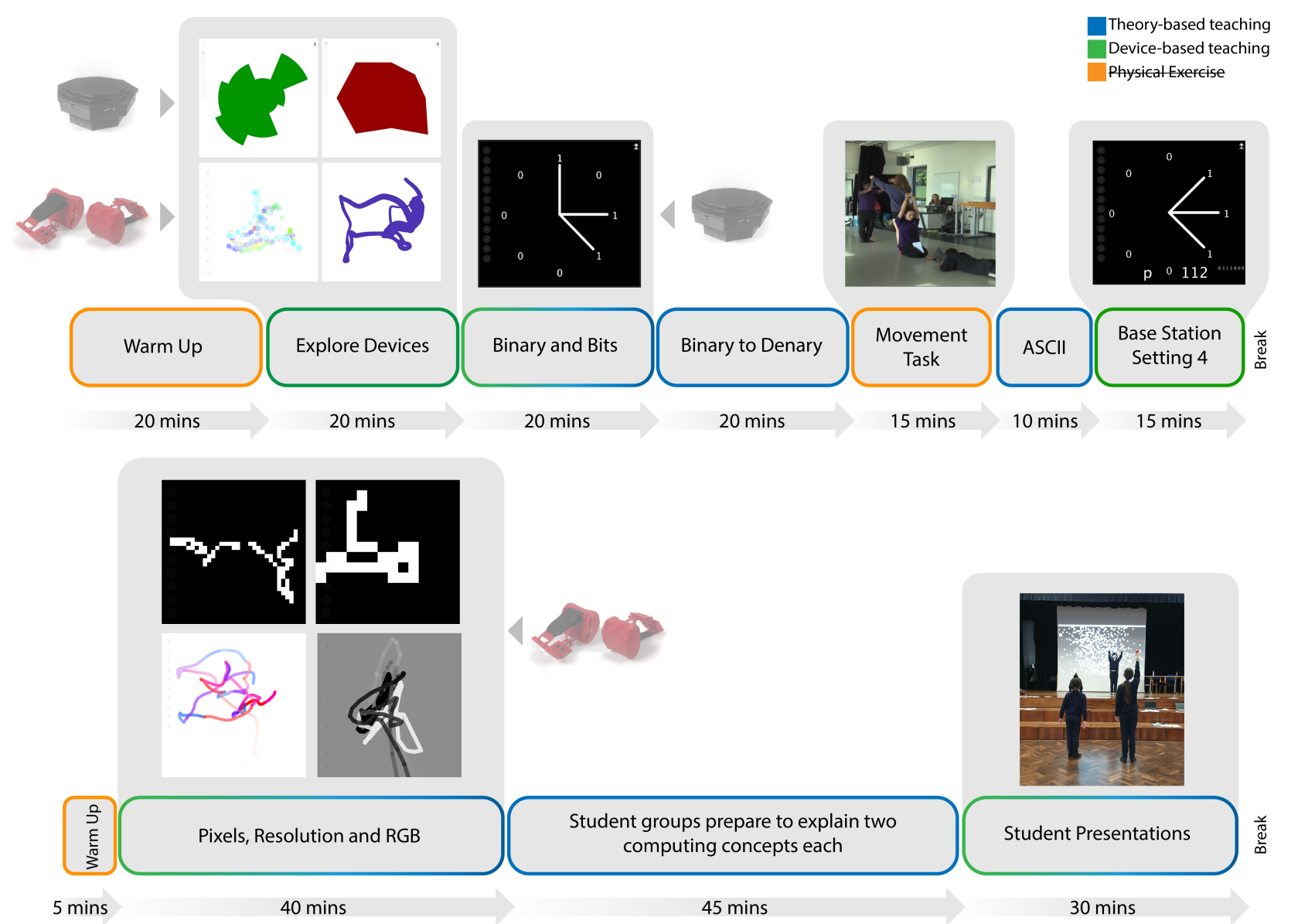

Figure 8: A visual representation of the workshop structure and topics covered.

analysis methods in more detail.

\section{Observation notes}

One researcher led the collection of observation notes throughout both workshops. In addition to the observer researcher, C, D, and one more researcher were present; they all conferred with the observer researcher their observations. It was agreed that the foci were to observe the extent that the DataMoves devices and the workshops as a whole, met the design criteria of: 1) enabling collaborative learning through group-based interaction, 2) enhancing learning through discovery and experimentation and 3) affording creativity. The notes comprised specific examples of how the students interacted with the DataMoves devices during the different learning activities they were asked to complete, along with observations of broader patterns of interaction (e.g., when the students seemed disengaged, or which activities they struggled with more). To support the observations, directly after each workshop, the observer researcher also gathered feedback from D and C who ran them, as well as the students' teachers, who were present for parts of the workshops. After the workshop, the observation notes collected were discussed within the whole team and expanded into long form written accounts. The instances contained within these written accounts, were then arranged in line with the three analytic foci.

\section{Interviews held with a subset of the students after the workshops}

A subset (a total of 6) of the participating students from the workshops was selected at random to be interviewed about their experiences, directly after the workshops had finished. All of the interviews were semi structured and focused on asking the students to reflect on their overall experiences of the workshop and their experiences of using the DataMoves devices. The students were also asked about how well they felt they understood the number and data concepts that were presented throughout the day, and which aspects of the workshop were most or least helpful in this respect. Finally, they were asked to reflect on whether and how their perceptions of computing as a subject changed as a result of the workshop. The interviews were recorded, transcribed and analysed using thematic analysis. 


\subsection{Findings}

Overall, the findings demonstrate how the combination of the movement-based activities, with and without the DataMoves devices, was successful at promoting a learning experience that was both engaging and challenging to the students. Our observations of how the learning activities took place when interacting with the DataMoves devices, demonstrated that the devices coupled with the visualisations triggered much discussion amongst the students about the concepts of binary, ASCII, pixels and resolution. These discussions were rooted in both discovering how to use the DataMoves devices to express a given binary number, ASCII character or resolution and in deciding how to best convey a concept when performing it to their peers. Another positive finding in both workshops was how inclusive they were: even the students who self-reported themselves as less confident in their computing skills when asked about them during the informal, introductory activity at the beginning of the workshop were found to actively contribute to creating choreographies within their teams and to volunteer to answer the instructors' questions about number systems and data representation, within a short time of starting the workshop. Moreover, based on the interviews, the students felt that the workshop improved their understanding of both the topics of number systems and data representation. All six of the students who were interviewed reported that both the DataMoves devices and the movement-based learning activities helped them understand the topics much better than they had previously.

Next, we describe in more detail some of the patterns of interaction observed, and how they contributed to developing the students' understanding of number systems and data representation.

6.1.1 Did the DataMoves devices enable collaborative learning? A key difference between the base station and the wearable was the extent to which they were designed to support individual and group interaction: the base station was designed to afford up to 8 students interacting with it at one time, while the wearable was designed to be worn by one person at a time. Despite this, we found that both of the devices were able to support collaborative learning, especially in terms of prompting the students to discuss together how their sensed movements mapped onto the visualisations. For the base station, the group interaction unfolded as expected - when engaging in group activities, the students actively discussed the learning activities together and worked as a team to coordinate their movements to elicit a particular visualisation to be created (e.g., a binary number or ASCII character). Within groups, they directed each other to stand in physical points in space, using the projected visualisation to reflect on the underlying concepts, for example the directionality of reading binary.

Surprisingly, even though the wearable was intended to be used by one person at a time, during the introductory part of the workshop, where the students were able to use it without being instructed, we found much evidence of unprompted collaboration taking place. All of the students actively shared it, by way of taking turns to wear and experiment with it, and actively watching on and discussing output when others in their group were using it. Strikingly, rather than quietly observing the visualisation, in all instances the students actively discussed the visualisations that were being created together, instructing each other with ideas of how to make specific shapes and colours. This seemed to be afforded by the output displayed on the large, shared projection, and by the visualisations themselves which triggered the students' interest. The two groups that chose to incorporate the wearable into their choreography task at the end of the workshop, also both created routines where they mimicked and mirrored each other's movements, giving the impression that all their movements were contributing to the visualisation being generated on the wall display (see Figure 1).

6.1.2 How did the exploration and discovery afforded by the DataMoves devices, contribute to learning? In addition, during the initial part of the workshop, where the students were able to openly experiment with both the base station and the wearables, we observed a high level of excitement and curiosity. The students tried out all of the available visualisations when using both devices. They were very vocal and talked to each other, generating hypotheses with their peers about what the devices were sensing, and how different movements mapped to the visualisations that were displayed.

\section{Movement-based exploration as a means of "debugging" under- standing}

It was noted how some of the mappings between their movements and the visualisations were not immediately obvious to the students; for example, it took the students some time to figure out how far to step away from the base station, or how low to crouch down, to change the bit value that was displayed from a " 1 " to a "0". Equally, they initially indicated some uncertainty about how the wearable mapped their movement data. Because the wearable mapped 3D movement to a 2D visualisation, it did not display their movement in space directly, as they had expected. When faced with these unexpected forms of output, the students switched between discussing them within their groups, and asking the instructors for help. In the interviews, they also noted how the mappings between their physical movements and the visualisations were sometimes ambiguous, but that this was what spurred them on to work out how the devices worked, and to reflect on underlying concepts. In one example, S4 described how figuring out the mapping between her peers' movements and the visualisations from the base station helped her realize that up to that point, she had been reading binary backwards. In another, S2 discussed the mappings between $3 \mathrm{D}$ movement and the $2 \mathrm{D}$ visualisations when using the wearable device: "I really liked the bracelet thing because you had to use a lot of thinking to work it out." In sum, rather than see this as frustrating they relished the challenge of fathoming how the devices and visualisations worked together.

\section{Open-ended exploration as a precursor to structured activities}

Hence, engaging with open-ended exploration of the DataMoves devices was found to help the students develop an initial understanding of the input and output. In turn, it enabled them to focus on subsequent task-specific goals when using the DataMoves devices again later in the session. For example, where they were asked to form an 8-bit ASCII character with the base station as a group, they immediately started testing out movements that they had already 
worked out would cause a switch between 1s and 0s. Instead of having to focus on how the technology worked, they were able to focus on deciding which group members would "enact" $1 \mathrm{~s}$ and $0 \mathrm{~s}$, on coming up with creative movements and on instructing each other.

\section{Exploration as a catalyst for discussion}

We also observed throughout the workshop, that the embodied nature of the DataMoves devices, elicited a high level of discussion amongst the students about the concepts being taught. For instance, when working together as a group to create binary representations of ASCII characters, they were found to discuss, unprompted, the direction in which bytes are read, while concurrently experimenting with different ways of physically arranging themselves around the base station to form the intended string of binary digits.

Asking the students to perform and explain their thought process to others for the creative tasks they were set, also triggered reflection and discussion, enabling them to practice and talk about number systems in different ways than is typical in the classroom. In particular, they spent time talking and contesting with each other about how to convey the concept to others, deciding on the best movements to use, performing to the instructors and asking for feedback from them. While not all of the time was spent discussing the target concepts (much time was spent just figuring out the choreography), they were able to go between experimenting with group movements and the DataMoves devices to better understand number systems while using the tools to put together their performances.

In sum, the learning activities with the DataMoves devices were found to be able to deepen their understanding of the topics covered in the workshop. In some cases, the activities were able to help them to clarify their understanding. For example, S1 discussed how "I had confusion as to how people used binary numbers and converted them and then how computers would understand those numbers and so on but after using the [DataMoves] devices for example I saw how the sensors could detect people around them and tell the distance between the actual object and so on and I saw how the binary numbers changed and that's how I learned more". For others, the movement-based activities served as a way of helping them better remember the topics. For instance, S4 discussed how making up a dance routine to demonstrate her understanding of denary to the rest of the class "was quite fun, that helped denary stick in my head a bit more."

6.1.3 Were the students able to creatively appropriate the DataMoves devices? Facilitating creativity was a design strategy built into both the workshop structure and into the DataMoves devices. In terms of the workshop, the students were asked to work in groups on a creative, movement-based task to present back to their peers. From the device perspective, the aim was to facilitate creative, embodied interaction, through making a variety of visualisations available to the students and designing the sensor-based devices in such a way that they could experiment with a wide range of embodied movements. These affordances legitimised and resulted in much creative interaction during the workshops. The student groups came up with many uses coupling the visualisations and devices to "perform" one of the number concepts they had learned during the day to the whole class. For example, one group of four decided to embody moving sculptures around the base station, creating ASCII characters through coordinated movement, and freezing in place for each letter. For the wearables, two of the groups in workshop B decided to demonstrate how color depth complexifies when the number of bits is increased. Both of these groups started with a simple choreography involving two movements, and then built up to a coordinated and complex dance - with one member switching the corresponding software to display more and more complex visualisations as the movements became more complex. Two of the groups also chose to use the DataMoves devices as a more decorative aspect to support their choreographies, playing the changing visualisations in the background, but not foregrounding them as part of their performance. In sum, the students were highly creative and playful when using the DataMoves devices; coming up with a diversity of embodied movements to control them, exploring a range of visualisations to represent a particular concept and utilising the devices to enhance the aesthetics of their choreography.

6.1.4 The experiential and pedagogical value of the DataMoves devices. The students who were interviewed described how learning about number systems and data representation in this way was surprisingly "fun". Their responses indicated that two aspects of the workshop, in particular, were tied to the level of enjoyment that they experienced: (i) the creativity afforded by the tasks they were asked to engage with, and (ii) the opportunities that the workshop provided for them to move and dance. In particular, they mentioned how much they enjoyed the color mixing activity using the wearable and the opportunity to create their own choreography.

For example, S3 said the workshop was more enjoyable than the typical computing lesson that requires "sitting all day", noting, "I liked how today we got to learn in a different way. I really enjoyed the movement element. It helped me remember better other than just writing down in a book." Another remarked on how the movement and dance made the aspects of computing taught "more interesting than sitting at a desk practicing with books or computers" [S1]. When asked to reflect about their experiences using the DataMoves devices, specifically, the interviewed students provided overwhelmingly positive feedback. They described the DataMoves devices as "cool" [S5] and "fun" [S6].

When asked what they thought about computer science, three of the six participants explicitly referred to the workshop as changing their perception of computing from "dull" to "fun" or "interesting." For instance, S4 noted how "I used to think Computer Science was just the dullest subject, just talked about computers but now it's showing me that there's so many different things you can make." Two participants referred to the change in their perception as resulting from gaining a deeper understanding of the topics covered (i.e., binary number systems and related concepts). For example, S1 said, "normally when I don't understand something properly then I leave it because I can't do it properly but because my understanding on it is slightly broader than before I'm more attracted to it and I feel I should explore a bit more." 
The teachers, on the other hand, commented on the interdisciplinary value of the DataMoves workshop: "It shows people that dance isn't just about dancing - it also connects to lots of other subject areas" [T1]. Another teacher noted that our more creative approach to teaching computing "lends itself to a wide range of performing arts subjects, and even humanities subjects, maths - it's really important", further adding "the more initiatives like this, the better" [T2].

\section{DISCUSSION}

One of the main findings from the DataMoves project was to demonstrate how computer science topics that are often perceived as dull and boring to learn (and teach) can be brought alive and made exciting and fun through a creative use of paper-based materials, physical artefacts, technologies and learning activities. Here we showed how ideating a computing workshop with open-ended physical computing prototypes enabled us to explore new ways of grounding the abstract concepts of number systems and data representation; we did this by coupling them with movement and choreography activities, while extending the repertoire of what is normally carried out in dance and computing workshops. It allowed for playful forays in how to innovate learning, moving from simply covering the curriculum to eliciting more curious, creative and collaborative ways by which to both teach and learn. However, rather than let the novel technology take centre stage, and be viewed as the game-changer, it was designed to be more low-key; to be used to try out at different points in the learning process, make connections and to provide opportunities for unique learning experiences. Hence, we deliberately chose not to evaluate the efficacy of the tools per se to improve learning about computing concepts but to provide them as tools to be used as part of a medley of learning activities. Other times, during the workshop, the use of paper-based artefacts was seen by the workshop leaders (D and C) as being just as important at providing ways of expressing or articulating how a number system, like denary, works. This way of viewing technology resonates with Bekker et al's framework on using digital toolkits to promote the teaching of digital literacy and design thinking to school children by getting them to understand and experiment with a variety of materials and technological devices [8] They also suggest that children should not only learn technology skills (e.g. programming and building electronic circuits), but also should consider and discuss the value of such technologies in society. While the aim of our workshops did not go this far, we can say they were designed to enable the students to learn about the value and reasons for using different number systems in computing and creativity - which is something they do not usually experience in the curriculum.

Exploring new ways of learning about computer science concepts using technology in this way was achieved largely by having the DataMoves tools be part of a bricolage available when organising the workshop. In particular, it enabled us to craft them in ways we felt fit, so that students were free to enact out different number systems and ways of representing data. In other words, the pervasive computing technology was viewed as a resource to be used in creative ways to deepen the learning, through enacting out, and reflective understanding. Our partners from the dance and education sectors, also commented on how helping to design and play with the evolving prototypes enabled them to break out of their tried and tested ways of thinking about how to teach computing, transporting them into uncharted waters. In particular, they found new ways of dovetailing movement activities with teaching activities, such that they appeared to be more seamless and interwoven. Beforehand, they had been set up to be separate activities, where dancing was seen more as energizing, or as a form of ice-breaking, and distinct from the coding. In the new workshop, the moving and dancing activities took place throughout, being integrated more with the computing learning activities.

We also found that providing two different kinds of devices enabled different forms of creativity, exploration and collaboration to take place. The wearable encouraged turn-taking, with the wearer trying out different resolution and color visualisations when moving their arm around, and the others looking on and making suggestions about what to do next, while providing feedback on the different movements they were making. The base station allowed the students to work in groups, mapping their movements with the number system concepts being explored. The sometimes ambiguous visualisations, which the students had to work out through experimenting with their body movements, were found to be both challenging and intriguing, bringing enthusiasm to the learning of concepts they might have otherwise considered dull. Moving in and out of the sensing zones around the base station, while watching their collective visualisations appear provided them with different perspectives and the opportunity to step in and out [2] of their learning and understanding. Asking groups to create their own binary number sequences as a dance performance was also viewed as a highly successful learning method, enabling the students to create, compare and comment on each other's work.

We also discovered that the technology does not always have to be the focal point but that it can be brought to the fore at opportune times and then placed aside while other learning activities take place. This approach contrasts with our earlier work on designing and using tangibles to learn about computing, where the classroom activities were motivated by experimenting primarily with the new interactive technology for shorter periods of time, like a typical school lesson. For example, our research on using physical computing devices for teenagers in a 6-week course on computing, using our Magic Cubes [26] showed how students used them in a variety of ways to explore their environment while coding them to perform certain actions - hence, for the most part they were always in their hands. This proved to be a highly effective strategy for collaborative learning for weekly 90 -minute classes. Here, our wearables and base station were used more judiciously in a full day workshop setting which, it turned out to be highly effective. The students understood their role and did not expect them to do more. We also learnt that 'less is more' in this setting; in the sense of both implementing minimal functionality to trigger imagination and reflection and keeping the role of the technology to be relatively limited in a diverse and multi-activity workshop.

Finally, we observed how our new kind of free-flowing, openended and challenging workshop encouraged more active engagement from all students - not just the keen ones, eager to show their computing prowess but also those who may have had less confidence in their computing skills or have previously considered 
the subject to be dull. Here they blossomed and were able to actively take part without being too self-conscious - whether it was dancing or computing. The comments made by the students were witness to this refreshing take on inclusivity.

\subsection{Future work}

Like many projects, our research was curtailed by the sudden onset of COVID-19. However, we were fortunate to have been able to run the project throughout 2019 which cumulated in conducting two full-day workshops. Our plan was to conduct a series of further workshops throughout 2020 to scale the approach up and then offer it to schools throughout the UK. This is now future work. We have also been exploring how we can expand the material repertoire of the base station; for example, we have begun experimenting with a soundscape interface, transforming it into an instrument where every activated sensor can make a unique sound. For the wearables, we are considering how more varied visualisations could be created that could be used to learn about other topics, such as Physics, including concepts such as speed and acceleration using the integrated IMU on the physical computing device, which in return would draw graphs in real-time.

\section{CONCLUSIONS}

Our approach to rethinking how to teach computer science concepts, such as computation and data, demonstrates how they can be learnt in a meaningful and playful way by being experienced through physical movement and exploration. Our approach also demonstrates how novel technology, in the form of physical computing devices, is just one of many materials that can be used to create an exciting, challenging, and enjoyable learning experience. The two workshops we ran showed how abstract and perceived-tobe dull topics, like number systems, binary and pixels can be taught in fun, stimulating and challenging ways. Our findings showed a high level of student engagement, suggesting that our novel design approach can not only improve students' understanding of the computing topics, but also provide inspiration for teachers about how to teach them. In so doing, we have demonstrated how to design physical computing devices for quite different purposes compared with only being used as a vehicle to learn to code.

\section{ACKNOWLEDGMENTS}

This research has been made possible by the EPSRC IAA Knowledge Exchange Dance and Coding Project (IAA 2017-20) and the AHRC Creative Informatics Project (AH/S002782/1). We would like to give a profound thanks to our collaborators for their time, insight and enthusiasm, as well as thank all of the children who participated in the workshop deployments. We would also like to thank Jo Townshend from UCL and Jasmine Wilson of Studio Wayne McGregor for their invaluable contributions to this study.

\section{REFERENCES}

[1] Dor Abrahamson, Alejandro Andrade, Arthur Bakker, Mitchell J Nathan, Candace Walkington, Robb Lindgren, David E Brown, Asnat R Zohar, Sharona T Levy, Joshua A Danish, and Others. 2018. Moving forward: In search of synergy across diverse views on the role of physical movement in design for stem education In Proceedings of International Conference of the Learning Sciences, ICLS, Vol. 2. International Society of the Learning Sciences, 1243-1250.
[2] Edith Ackermann. 1996. Perspective-taking and object construction: Two keys to learning. Constructionism in practice: designing, thinking, and learning in a digital world, Lawrence Erlbaum, Mahwah, Nf (1996), 25-35.

[3] Neil Anderson, Colin Lankshear, Carolyn Timms, and Lyn Courtney. 2008. 'Because it's boring, irrelevant and I don't like computers': Why high school girls avoid professionally-oriented ICT subjects. Computers and Education 50, 4 (2008), 1304-1318. https://doi.org/10.1016/j.compedu.2006.12.003

[4] Alissa N. Antle. 2009. Embodied child computer interaction: Why embodiment matters. Interactions 16, 2 (2009), 27-30. https://doi.org/10.1145/1487632.1487639

[5] Alissa N Antle. 2013. Research opportunities: Embodied child-computer interaction. International fournal of Child-Computer Interaction 1, 1 (2013), 30-36. https://doi.org/10.1016/j.ijcci.2012.08.001

[6] Massimo Banzi. 2014. Getting Started with Arduino (3rd ed.). Maker Media. 262 pages.

[7] Angelos Barmpoutis, Qianwen Ding, Lisa Anthony, Wanda Eugene, and Marko Suvajdzic. [n.d.]. Exploration of kinesthetic gaming for enhancing elementary math education using culturally responsive teaching methodologies. In 2016 IEEE Virtual Reality Workshop on K-12 Embodied Learning through Virtual \& Augmented Reality (KELVAR). IEEE, 1-4. https://doi.org/10.1109/KELVAR.2016.7563674

[8] Tilde Bekker, Saskia Bakker, Iris Douma, Janneke Van Der Poel, and Koen Scheltenaar. 2015. Teaching children digital literacy through design-based learning with digital toolkits in schools. International fournal of Child-Computer Interaction 5 (2015), 29-38. https://doi.org/10.1016/j.ijcci.2015.12.001

[9] Tilde Bekker, Janienke Sturm, and Berry Eggen. 2010. Designing playful interactions for social interaction and physical play. Personal and Ubiquitous Computing 14, 5 (2010), 385-396. https://doi.org/10.1007/s00779-009-0264-1

[10] Paulo Blikstein. 2013. Digital fabrication and 'making'in education: The democratization of invention. FabLabs: Of machines, makers and inventors 4, 1 (2013), 1-21. https://doi.org/10.14361/transcript.9783839423820.203

[11] Paulo Blikstein. 2013. Gears of our Childhood: Constructionist toolkits, robotics, and physical computing, past and future. ACM International Conference Proceeding Series (2013), 173-182. https://doi.org/10.1145/2485760.2485786

[12] Neil C.C. Brown, Sue Sentance, Tom Crick, and Simon Humphreys. 2014. Restart: The resurgence of computer science in UK schools. ACM Transactions on Computing Education 14, 2 (2014), 1-22. https://doi.org/10.1145/2602484

[13] Kayla DesPortes, Monet Spells, and Betsy DiSalvo. 2016. Interdisciplinary Computing and the Emergence of Boundary Objects: A Case-Study of Dance and Technology. Singapore: International Society of the Learning Sciences.

[14] Paul Dourish. 2004. Where the action is: the foundations of embodied interaction. MIT press.

[15] Paul Dourish. 2017. The Stuff of Bits: An Essay on the Materialities of Information. The MIT Press.

[16] Michail N. Giannakos, Letizia Jaccheri, and Roberta Proto. 2013. Teaching Computer Science to Young Children through Creativity: Lessons Learned from the Case of Norway. In Proceedings of the 3rd Computer Science Education Research Conference on Computer Science Education Research (Arnhem, Netherlands) (CSERC '13). Open Universiteit, Heerlen, Heerlen, NLD, 103-111. https://doi.org/10.5555/2541917.2541927

[17] Anuradha A. Gokhale. 1995. Collaborative Learning Enhances Critical Thinking. Journal of Technology Education 7, 1 (1995), 22-30. https://doi.org/10.21061/jte. v7i1.a. 2

[18] Tom Hitron, Idan David, Netta Ofer, Andrey Grishko, Iddo Yehoshua Wald, Hadas Erel, and Oren Zuckerman. 2018. Digital Outdoor play: Benefits and risks from an interaction design perspective. In Proceedings of the 2018 CHI Conference on Human Factors in Computing Systems. 1-13. https://doi.org/10.1145/3173574. 3173858

[19] Tom Hitron, Yoav Orlev, Iddo Wald, Ariel Shamir, Hadas Erel, and Oren Zuckerman. 2019. Can children understand machine learning concepts? The effect of uncovering black boxes. Conference on Human Factors in Computing Systems Proceedings (2019), 1-11. https://doi.org/10.1145/3290605.3300645

[20] Michael S Horn and Robert J K Jacob. 2007. Tangible Programming in the Classroom with Tern. In CHI '07 Extended Abstracts on Human Factors in Computing Systems (CHI EA '07). Association for Computing Machinery, New York, 19651970. https://doi.org/10.1145/1240866.1240933

[21] Peter Hubwieser, Michail N. Giannakos, Marc Berges, Torsten Brinda, Ira Diethelm, Johannes Magenheim, Yogendra Pal, Jana Jackova, and Egle Jasute. 2015. A global snapshot of computer science education in K-12 schools. ITiCSE-WGP 2015 - Proceedings of the 2015 ITiCSE Conference on Working Group Reports (2015), 65-83. https://doi.org/10.1145/2858796.2858799

[22] Robert J K Jacob, Audrey Girouard, Leanne M Hirshfield, Michael S Horn, Orit Shaer, Erin Treacy Solovey, and Jamie Zigelbaum. 2008. Reality-Based Interaction: A Framework for Post-WIMP Interfaces. In Proceedings of the SIGCHI Conference on Human Factors in Computing Systems (CHI '08). Association for Computing Machinery, New York, 201-210. https://doi.org/10.1145/1357054.1357089

[23] Yasmin B Kafai, Kylie A Peppler, Quinn Burke, Michael Moore, and Diane Glosson. 2010. Fröbel's forgotten gift: textile construction kits as pathways into play, design and computation. In Proceedings of the 9th International Conference on Interaction Design and Children. 214-217. https://doi.org/10.1145/1810543.1810574 
[24] Susan Lechelt, Yvonne Rogers, and Nicolai Marquardt. 2020. Coming to your senses: Promoting critical thinking about sensors through playful interaction in classrooms. Proceedings of the Interaction Design and Children Conference, IDC 2020 (2020), 11-22. https://doi.org/10.1145/3392063.3394401

[25] Zuzanna Lechelt, Yvonne Rogers, Nicolai Marquardt, and Frederick Brudy. 2017. MakeMe, CodeMe, ConnectUs: Learning digital fluency through tangible Magic Cubes Abstract. In ETIS 2017: 3rd European Tangible Interaction Studio.

[26] Zuzanna Lechelt, Yvonne Rogers, Nicola Yuill, Lena Nagl, Grazia Ragone, and Nicolai Marquardt. 2018. Inclusive computing in special needs classrooms: Designing for all. In Proceedings of the 2018 CHI Conference on Human Factors in Computing Systems. 1-12. https://doi.org/10.1145/3173574.3174091

[27] Eunkyoung Lee, Yasmin Kafai, Veena Vasudevan, and Richard Davis. 2014. Playing in the Arcade: Designing Tangible Interfaces with MaKey MaKey for Scratch Games. 277-292. https://doi.org/10.1007/978-981-4560-96-2_13

[28] Victor R. Lee and Joel Drake. 2013. Quantified recess: Design of an activity for elementary students involving analyses of their own movement data. ACM International Conference Proceeding Series Idc (2013), 273-276. https://doi.org/10. $1145 / 2485760.2485822$

[29] Robb Lindgren and Mina Johnson-Glenberg. 2013. Emboldened by embodiment Six precepts for research on embodied learning and mixed reality. Educational researcher 42, 8 (2013), 445-452. https://doi.org/10.3102/0013189X13511661

[30] Edward Melcer. 2017. Moving to Learn: Exploring the Impact of Physical Embodiment in Educational Programming Games. In Proceedings of the $2017 \mathrm{CHI}$ Conference Extended Abstracts on Human Factors in Computing Systems (CHI EA '17). Association for Computing Machinery, New York, NY, USA, 301-306. https://doi.org/10.1145/3027063.3027129

[31] Netta Ofer, Idan David, Hadas Erel, and Oren Zuckerman. 2019. Coding for outdoor play: A coding platform for children to invent and enhance outdoor play experiences. Conference on Human Factors in Computing Systems - Proceedings (2019). https://doi.org/10.1145/3290605.3300394

[32] Sara Price and Yvonne Rogers. 2004. Let's get physical: The learning benefits of interacting in digitally augmented physical spaces. Computers \& Education 43 1-2 (2004), 137-151. https://doi.org/10.1016/j.compedu.2003.12.009

[33] Jessica Roberts, Leilah Lyons, Francesco Cafaro, and Rebecca Eydt. 2014. Interpreting data from within: Supporting humandata interaction in museum exhibits through perspective taking. In Proceedings of the 2014 conference on Interaction design and children. 7-16. https://doi.org/10.1145/2593968.2593974
[34] Yvonne Rogers, William R Hazlewood, Paul Marshall, Nick Dalton, and Susanna Hertrich. 2010. Ambient Influence: Can Twinkly Lights Lure and Abstract Representations Trigger Behavioral Change?. In Proceedings of the 12th ACM International Conference on Ubiquitous Computing (UbiComp '10). Association for Computing Machinery, New York, 261-270. https://doi.org/10.1145/1864349.1864372

[35] Y. Rogers, S. Price, G. Fitzpatrick, R. Fleck, E. Harris, H. Smith, C. Randell, H. Muller, C. O'malley, D. Stanton, M. Thompson, and M. Weal. 2004. Ambient wood: Designing new forms of digital augmentation for learning outdoors. Proceedings of the 2004 Conference on Interaction Design and Children: Building a Community, IDC 2004 (2004), 3-10. https://doi.org/10.1145/1017833.1017834

[36] Yvonne Rogers, Venus Shum, Nicolai Marquardt, Susan Lechelt, Rose Johnson, Howard Baker, and Matt Davies. 2017. From the BBC Micro to micro: Bit and beyond: A British innovation. Interactions 24, 2 (2017), 74-77. https://doi.org/10. 1145/3029601

[37] Yvonne Rogers, Ian Taylor, Danae Stanton, Claire O'Malley, Greta Corke, Silvia Gabrielli, Mike Scaife, Eric Harris, Ted Phelps, Sara Price, Hilary Smith, Henk Muller, Cliff Randell, and Andrew Moss. 2002. Things aren't what they seem to be. (2002), 373. https://doi.org/10.1145/778757.778766

[38] Daniela K. Rosner. 2012. The material practices of collaboration. Proceedings of the ACM Conference on Computer Supported Cooperative Work, CSCW (2012), 1155-1164. https://doi.org/10.1145/2145204.2145375

[39] Sue Sentence, Jane Waite, Steve Hodges, Emily Macleod, and Lucy Yeomans. 2017. Creating cool stuff" - Pupils' experience of the BBC micro:bit. Proceedings of the Conference on Integrating Technology into Computer Science Education, ITiCSE September 2014 (2017), 531-536. https://doi.org/10.1145/3017680.3017749

[40] Tiffanie R Smith and Juan E Gilbert. 2018. Dancing to design: a gesture elicitation study. In Proceedings of the 17th ACM Conference on Interaction Design and Children. 638-643. https://doi.org/10.1145/3202185.3210790

[41] Emilia Sobolewska. 2017. Tailoring methodological bricolage to investigate nondiscretionary use of digital technology. Electronic Visualisation and the Arts (EVA 2017) (2017), 1-11. https://doi.org/10.14236/ewic/HCI2017.49

[42] Anna Vallg \rarda and Ylva Fernaeus. 2015. Interaction Design as a Bricolage Practice. In Proceedings of the Ninth International Conference on Tangible, Embedded, and Embodied Interaction (TEI '15). Association for Computing Machinery, New York, 173-180. https://doi.org/10.1145/2677199.2680594

[43] Benjamin Wohl, Barry Porter, and Sarah Clinch. 2015. Teaching computer science to 5-7 year-Olds: An initial study with scratch, Cubelets and unplugged computing. ACM International Conference Proceeding Series 09-11-Nove (2015), 55-60. https://doi.org/10.1145/2818314:2818340 